
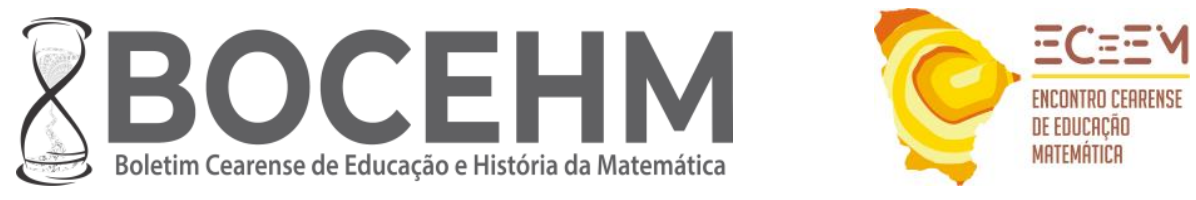

ISSN: $2447-8504$

DOI: $10.30938 /$ bocehm.v8i23.4964

(ब) $\odot \Theta$

\title{
UM ESTUDO DA PRIMEIRA E SEGUNDA PARTE DO TRATADO DA CIRCUNFERÊNCIA DE AL-KĀSHĪ
}

\section{A STUDY OF THE FIRST AND SECOND PART OF THE AL-KĀSHĪ CIRCUMFERENCE TREATY}

\author{
Kaline França Correia Andrade ${ }^{1}$
}

\begin{abstract}
RESUMO
O presente trabalho é um estudo sobre a primeira e a segunda parte do Tratado da Circunferência (al-Risāla al-Muhìtīyya) (1424). Este tratado foi escrito originalmente em árabe pelo astrônomo e matemático islâmico Ghiyāth al-Dīn Jamshīd ibn Mas'ūd ibn Mahmood al-Tabīb al-Kāshī (1380-1429), ou simplesmente al-Kāshī. O objetivo desse tratado era obter uma melhor aproximação da relação entre o comprimento da circunferência e o seu diâmetro; sua motivação veio da necessidade de maior precisão nas tabelas astronômicas devido aos avanços dos estudos astronômicos. Especificamente neste trabalho, apresentamos um estudo de parte do Tratado da Cicunferência que compreende um trecho da primeira parte e a segunda parte. O título da primeira parte do tratado é "Sobre a determinação da corda de um arco, que é a soma de um arco de uma corda desconhecida e do arco que é a metade de seu suplementar" e o título da segunda parte é "sobre a determinação do perímetro de um polígono qualquer, inscrito no círculo e do perímetro do semelhante circunscrito ao círculo". Assim, para o desenvolvimento deste trabalho, tomamos por base Azarian (2010) e a tradução do russo para o português de al-Kāshī (1954) que está ainda em andamendo. Além de apresentar o Tratado da Circunferência, faremos o passo a passo percorrido por al-Kāshī para a contrução de seu método recursivo para o cálculo do perímetro do polígono regular inscrito de $3.2^{n}$ lados e seu semelhante circunscrito, para finalmente obter a aproximação do comprimento da circunferência. Uma conclusão imediata é que relação entre essas duas partes se tornou fundamental para o desenvolvimento do tratado e, diante dos conhecimentos matemáticos mobilizados por al-Kāshī para atingir seus objetivos, concluímos que o Tratado da Circunferência é um documento histórico com potencialidades para elaboração de articulações entre história em ensino.
\end{abstract}

Palavras-chave: História da Matemática Medieval Islâmica; al-Kāshī; Tratado da Circunferência (al-Risāla al-Muhìtīyya).

\footnotetext{
ABSTRACT

The present work is a study on the first and the second part of the Circumference Treaty (al-Risāla al-Muhitìya) (1424). This treatise was originally written in Arabic by the Islamic astronomer and mathematician Ghiyāth al-Dīn Jamshīd ibn Mas'ūd ibn Mahmood al-Tabīb al-Kāshī (13801429), or simply al-Kāshī. The objective of this treaty was to obtain a better approximation of the relationship between the length of the circumference and its diameter; its motivation came from the need for greater precision in the astronomical tables due to the advances in astronomical

${ }^{1}$ Mestra em Matemática Aplicada e Estatística pela Universidade Federal do Rio Grande do Norte (PPGMAE/UFRN). Professora de Matemática do Instituto Federal de Educação, Ciência e Tecnologia do Rio Grande do Norte (IFRN), Natal, Rio Grande do Norte, Brasil. Endereço para correspondência: E-mail: kaline.andreza@hotmail.com.
}

(iD) ORCID iD: https://orcid.org/0000-0002-9237-2076. 
Kaline França Correia Andrade

Um estudo da primeira e segunda parte do tratado da circunferência de Al-Käshì

studies. Specifically in this work, we present a study of part of the Cicunference Treaty that comprises an excerpt from the first part and the second part. The title of the first part of the treaty is "On the determination of the string of an arc, which is the sum of an arc of an unknown string and the arc that is half of its supplementary" and the title of the second part is "on the determination of the perimeter of any polygon, inscribed in the circle and the perimeter of the similar circumscribed to the circle". Thus, for the development of this work, we used Azarian (2010) and the translation from Russian into Portuguese of al-Kāshī (1954), which is still in progress. In addition to presenting the Circumference Treaty, we will do the step-by-step covered by al-Kāshī for the construction of his recursive method for calculating the perimeter of the inscribed regular polygon of $3.2^{n}$ sides and its similar circumscribed, to finally obtain the approximation of the circumference length. An immediate conclusion is that the relationship between these two parties has become fundamental for the development of the treaty and, in view of the mathematical knowledge mobilized by al-Kāshī to achieve his objectives, we conclude that the Circumference Treaty is a historical document with potential for the elaboration of articulations between history in teaching.

Keywords: History of Medieval Islamic Mathematics; al-Kāshī; Circumference Treaty (al-Risāla al-Muhìtìya). 


\section{Introdução}

O tratado da Circunferência (al-Risāla al-Muhittīyya) (1424) é um trabalho marcante da matemática islâmica medieval, foi escrito em um contexto onde os estudos astronômicos ocupavam um lugar de destaque na ciência islâmica medieval e o seu avanço demandava tabelas astronômicas $(z \bar{j} j)$ cada vez mais precisas. Foi originalmente escrito em árabe e concluído em 1424 pelo matemático e astrônomo islâmico Ghiyāth alDīn Jamshīd Mas'ūd al-Kāshī, ou simplesmente al-Kāshī, (O’CONNOR; ROBERTSON, 1999), durante sua permanência em Samarcanda a convite do governante Ulugh Beg, para fazer parte da equipe de sábios de sua madrassa (tipo de universidade). Seu conteúdo é dedicado ao cálculo de uma melhor aproximação para a relação entre o comprimento da circunferência e o seu diâmetro, comparada às aproximações obtidas pelos seus predecessores: Arquimedes (287-212 a.C.), Abul'Wafā' al-Būzjān̄̄ (940-998) e Abū Rayhān al-Bīrūn̄̄ (973-1050).

A cópia de que dispomos para a elaboração desse trabalho consiste de uma tradução russa, al-Kāshī (1954), feita por Boris Rozenfeld e publicada na revista Pesquisa Histórica e Matemática (ИСТОРИКО-МАТЕМАТИЧЕСКИЕ ИССЛЕДОВАНИЯ Istorico-Matematicheskie Issledovaniya), edição $\mathrm{n}^{\circ}$ 07, uma publicação de Estado de literatura técnico-teórica, em Moscou, 1954, editada por G.F. Rybkina e A.N. Youschkevitch. Possui cinquenta e duas páginas e é divido em introdução, dez partes e conclusão. Deixamos claro que todas as demonstrações e relações matemáticas presentes no Tratado da Circunferência, foram feitas por extenso, com excessão das tabelas de cálculos.

O presente trabalho consiste de um estudo específico sobre um trecho da primeira parte do tratado (onde al-Kāshī desenvolve o que hoje podemos denominar de teorema) e da segunda parte, na qual al-Kāshī desenvolve um método recursivo para o cálculo das sucessivas cordas e, consequentemente, dos sucessivos lados dos polígonos regulares inscritos à circunferência, com vistas a obter o perímetro de um polígono de $3.2^{n}$ lados. Nesse processo, vamos também identifcar os conhecimentos matemáticos mobilizados por al-Kashi para alcançar seu objetivo. Adotando uma historiografia atualizada "que leva em conta a contextualização dos processos de elaboração, transmissão e adaptação de conceitos" (BELTRAN; SAITO; TRINDADE, 2014, p.15), este trabalho foi elaborado dentro da perpectiva que busca articular a história e o ensino de matemática, não somente 
Kaline França Correia Andrade

Um estudo da primeira e segunda parte do tratado da circunferência de Al-Kāshī

através da compreensão do processo de formação dos conhecimentos matemáticos dentro de seu contexto, mas também prima pelo conteúdo matemático em si, (SAITO; DIAS, 2013, p.96).

Dividimos nosso trabalho em introdução, três partes e conclusão: na parte 1, falamos sobre o objetivo do Tratado da Circunferência e o teorema criado por al- Kāshī; na parte 2, fizemos os cálculos das sucessivas cordas; na parte 3, contamos os lados do polígono de $3.2^{n}$ lados e, por último, tecemos nossas conclusões e considerações finais.

\section{Parte 1: o objetivo e teorema central}

$\mathrm{Na}$ introdução do tratado, após analisar as aproximações feitas por seus predecessores, Arquimedes (287-212), Abu'l-Wafā Būzjānī (940-998) e Abu Rayhān alBīrūnī (973-1048), e concluir que não eram satisfatórias, al-Kāshī estabelece um ousado objetivo:

[...] queremos determinar a circunferência do círculo com a suposição de que seu diâmetro é conhecido em termos de uma determinada medição de tal maneira que estaríamos certos de que em um círculo que seu diâmetro é seiscentas mil vezes maior que o diâmetro da esfera terrestre, a diferença entre o resultado de nossos cálculos e o valor real não ultrapasse a largura de um fio de cabelo, ou seja, um sexto da largura de um grão de cevada, de modo que tal diferença para círculo menor que este seja ainda menor. ${ }^{2}$ (AL-KĀSHĪ, 1954, p.329, tradução nossa).

Logo, al- Kāshī queria determinar o comprimento da circunferência de diâmetro conhecido em termos de alguma medição (ou seja, em alguma unidade de medida), de tal forma que, para um círculo de diâmetro seiscentas mil vezes maior que o diâmetro da Terra, a diferença entre o valor real e o valor calculado não fosse maior que a largura de um fio de cabelo de cavalo, que equivale a um sexto da largura de um grão de cevada; além disso, para círculos menores, essa margem de erro seria menor ainda.

Então, na primeira parte do tratado, embora não use essa denominação, al- Kāshī desenvolve o que hoje podemos denominar de teorema (Figura 1):

\footnotetext{
2““...] мы хотим так определить окружность круга в частях, в которых выражен диаметр, чтобы мы были уверены, что в круге, диаметр которого равен шестистам тысячам диаметров земли, разница между ней [полученной величиной окружности] и истинной была не больше волоса, т.е. одной шестой ширины среднего ячменного зерна, так что разность для круга, меньшего чем этот, была бы меньше" (AL-K $\bar{A} S H \bar{I}, 1954$, p.329, tradução nossa).
} 
Digo que: uma superfície [retangular] [construída] na soma do diâmetro e da corda de cada arco menor que o semicírculo e na metade do diâmetro [10] é igual ao quadrado da corda do arco que é igual à soma do primeiro arco e a metade de seu suplemento ao semicírculo ${ }^{3}$ (AL-KĀSHĪ, 1954, p.329, tradução nossa).

\section{Figura 1}

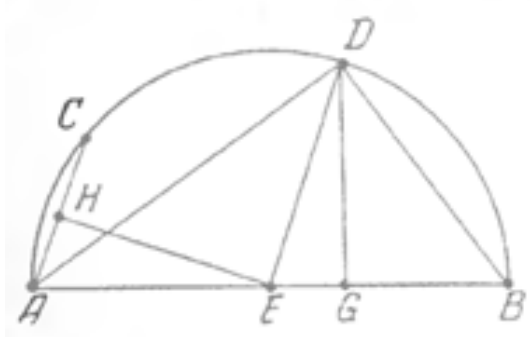

Fonte: al- Kāshī (1954, p.329).

Ou seja, dado um arco AD que é a soma de um arco conhecido AC com a metade de seu suplemento $C D$, o quadrado da sua corda $\overline{A D}$ é igual a superfície retangular construída na soma do diâmetro $A B$ e da corda desse arco menor que o semicírculo $A C$ e na metade do diâmetro $R$, onde $A B=120$. No contexto de al-Kāshī uma superfície retangular significava o produto entre dois valores, logo, escrevendo em notação moderna temos que $R .(A B+A C)=A D^{2}$. Em Azarian (2010) este teorema é denominado de teorema fundamental de al-Kāshī e é enunciado da seguinte forma:

(Teorema Fundamental de Kāshī). Se em um semicírculo com o diâmetro $A B=2 r$ e o centro E consideramos um arco arbitrário $\mathrm{AG}$, e chamamos o meio do arco $\mathrm{GB}$ de ponto $\mathrm{D}$, e desenhamos a corda $\mathrm{AD}$, então, $R \cdot(A B+A C)=A D^{2} .($ AZARIAN, $2010, \mathrm{p} .68)$

Abaixo, temos a tradução do trecho da primeira parte do tratado em que al-Kāshī demonstra sua proposição:

A fim de demonstrar construamos o semicírculo $\mathrm{ACB}$ de centro E na linha $\mathrm{AB}$;
traçamos uma corda arbitrária $\mathrm{AC}$; [o arco] $\mathrm{BC}$ que é o suplemento de $\mathrm{AC}$
dividimos ao meio encontrando o ponto $\mathrm{D}$; ligamos $\mathrm{A}$ e $\mathrm{D}$. Então a asserção
[diz]: a superfície na metade do diâmetro e a soma $\mathrm{AB}$ e $\mathrm{AC}$ é igual ao
quadrado AD.
Demonstração: Traçamos BD. Então o ângulo ADB é reto pela proposição
trinta do terceiro [livro] d'Os Elementos.
A seguir baixamos do ponto D a perpendicular DG à linha AB. Obtém-se os
triângulos DBG e DAG, semelhantes aos triangulo ADB pela oitava

3، Я говорю: [прямоугольная] поверхность, [построенная] ра сумме диаметра и хорды каждой дуги, меньшей полукруга, и на половине диаметра $\left[{ }^{10}\right]$, равна квадрату хорды дуги, равной сумме первой дуги и половины её дополнения до полукруга.” (AL-KĀSHİ, 1954, p.329, tradução nossa). 
proposição do [livro] seis d'Os Elementos. Por isso, o diâmetro AB está para $\mathrm{AD}$ assim como $\mathrm{AD}$ está para $\mathrm{AG}$ e pela proposição dezenove do [livro] sete dos Elementos, a superfície no diâmetro é igual ao quadrado de AD.

A seguir, pelo ponto $\mathrm{H}$ traçamos a perpendicular $\mathrm{HE}$ ao segmento AC. Então $\mathrm{H}$ será ponto médio de AC pela proposição três do terceiro livro de Os Elementos. Ligamos os pontos ED. Uma vez que a medida do ângulo BAC é metade do arco CB, a mesma do ângulo BED, estes dois ângulos [BAC e DEG] são iguais. Por isto o triângulo AHE e EGD são iguais, uma vez que eles têm os ângulos retos $\mathrm{H}$ e G, ângulos iguais em $\mathrm{E}, \mathrm{A}$ e lados iguais $\mathrm{AE}$ e ED. Portanto, o lado EG é igual ao lado AH, que é metade de AC e a superfície sobre $\mathrm{AG}$, ou seja, a soma da metade do diâmetro e $\mathrm{EG}$ é igual à metade de $\mathrm{AC}$ e sobre o diâmetro é igual ao quadrado de $\mathrm{AD}$. Uma vez que a superfície sobre uma linha e sobre a metade de outra é igual superfície sobre a metade da primeira e sobre a outra inteira, então a superfície sobre a soma do diâmetro e o dobre de EG, i.e., sobre a soma do diâmetro e AC e sobre a metade do diâmetro é igual ao quadrado de $\mathrm{AD}$. Isto é o queríamos [demonstrar]. (ALKĀSHĪ, 1954, p.329-330, tradução nossa)

Com base neste resultado, na segunda parte do tratado, al-Kāshī desenvolveu seu método recursivo de cálculo de cordas de arcos.

\section{Parte 2: o cálculo das sucessivas cordas}

A segunda parte do tratado da circunferência (Figura 2) tem por título: "Segunda parte sobre a determinação do perímetro de um polígono qualquer, inscrito no círculo e do perímetro semelhante circunscrito ao círculo". Nele, o primeiro passo de al-Kāshī é construir um semicírculo de diâmetro $\overline{A B}$ (Figura 3). e o arco $A C$ igual a um sexto da circunferência, ou seja, a medida do arco AC é $60^{\circ}$, logo, a corda AC é igual ao raio da circunferência, de acordo com a proposição quinze do quarto livro de Os Elementos de Euclides. Prosseguindo com a demonstração, al-Kāshī divide ao meio sucessivos arcos: $\mathrm{D}$ divide ao meio o arco $\mathrm{CB}$ (que é suplementar a $\mathrm{AC}$ ), G é divide ao meio o arco DB, J divide ao meio o arco GB, P divide ao meio o arco JB e assim por diante. Observemos que ele determina as metades dos sucessivos arcos de circunferência, mas em nenhum momento fala em "ponto médio" ou "bissetriz", apenas fala em "dividir o arco ao meio". 
Figura 2 -Trecho da Segunda parte do Tratado da Circunferência.

БТОРОИ РАЗДЕЛ

ОБ ОПРЕЛЕ.ТЕШП ПЕРНМЕТРА ПРОПЗВОТЫНОГО MНОГОУГОЛЬННКА, ВННСАНОГО В КРРУ, ПI НЕРНМЕТРА ПодоБного ЕМу многоугольникА, OHНСАHНГО ОКОТО ЕРУГА

Постронм па литит $A B$ полукруг $A C B$ с пентром $L$ и предположым, тто [гуга] $A C$-одна шестая окружности. Тогда хорда $A C$ равна половине диаметра по нятпадцатому претскжени четвертой [кити] «Начал» [17]. Затсм разделим пополам [лугу] $C B$, лвляющуюо дополнениом [лугі] $A C$ до полукруга, в $D$, [разделим пополам дуту] $D B$ в $G$, [.сугу] $G B$ в $/ /$ и так далее сколько угодно. liak мы указали в предыдущем разделе, благодаря [известной хор-

де] $A C$, становится известной и [хорда] $A D$, отстода становнтся пзвестной [хорда] $A G$, отсюда стаповится известної [хорда] $A H$ п так далес сколько угодно. Тешерь, ссли мы

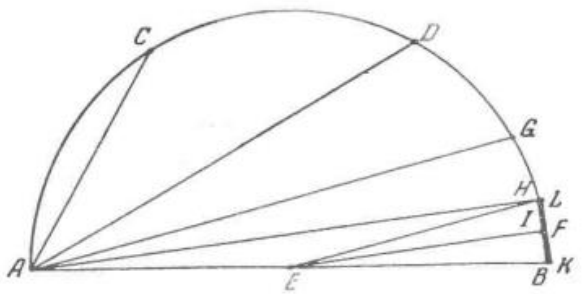

Fonte: al-Kāshī (1954, p.330-331).

\section{Figura 3}

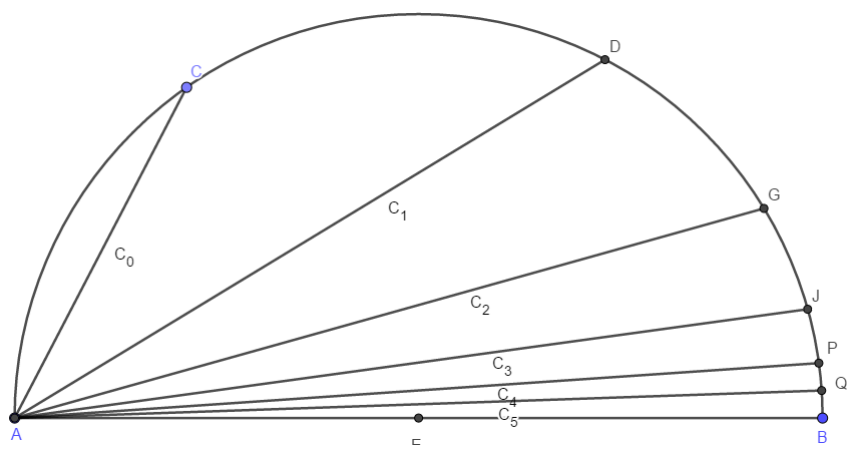

Fonte: Elaborado pela autora (2020).

Assim, utilizando o resultado da primeira parte do tratado: “[...] como conhecemos a corda $\mathrm{AC}$, também conheceremos a corda $\mathrm{AD}$, de onde também será conhecida a corda AG, AH, e assim por diante" (AL-KĀSHİ, 1954, p.30). Temos que, dado $R .(A B+A C)=A D^{2}$, obtemos:

$$
\begin{aligned}
& \text { R. }(A B+A C)=A D^{2} \\
& \text { R. }(A B+A D)=A G^{2} \\
& \text { R. }(A B+A G)=A J^{2}
\end{aligned}
$$


Generalizando, dado $R=60$ e $c_{0}=\sqrt{3}$ :

$$
\begin{aligned}
& \text { R. }\left(2 R+c_{n-1}\right)={c_{n}}^{2}, n \geq 1 \\
& c_{n}=\sqrt{R \cdot\left(2 R+c_{n-1}\right)}, n \geq 1
\end{aligned}
$$

Assim, al-Kāshī obtém um método recursivo para obter as sucessivas cordas $c_{n}$. Ele calcula seus valores através de vinte e oito tabelas desenvolvidas ao longo do tratado. Consideramos que, de fato, este o teorema central do tratado, pois todo ele se desenvolve a partir deste teorema.

Analisemos agora o seguinte trecho:

“[...] o ângulo AHB é reto pela proposição trinta do terceiro [livro] $<<$ d'OS Elementos $>$ [18] e, consequentemente, o quadrado de $\mathrm{AB}$ é igual aos quadrados AH e HB pelo "teorema da noiva"4 [19]" (AL-KĀSHĪ, 1954, p.331, tradução nossa).

Com a conclusão de que $A H B$ é um ângulo reto (Figura 4) pelo "Teorema da Noiva", que concluímos ser o que hoje denominamos de Teorema de Pitágoras, al-Kāsh̄̄ calcula a corda $H B$ corresponde às sucessivas cordas $a_{n}$ (Figura 5) que é o lado do polígono regular inscrito, assim, $H B=a_{n}=\sqrt{4 r^{2}-c_{n}^{2}}, n \geq 1$.

\section{Figura 4}

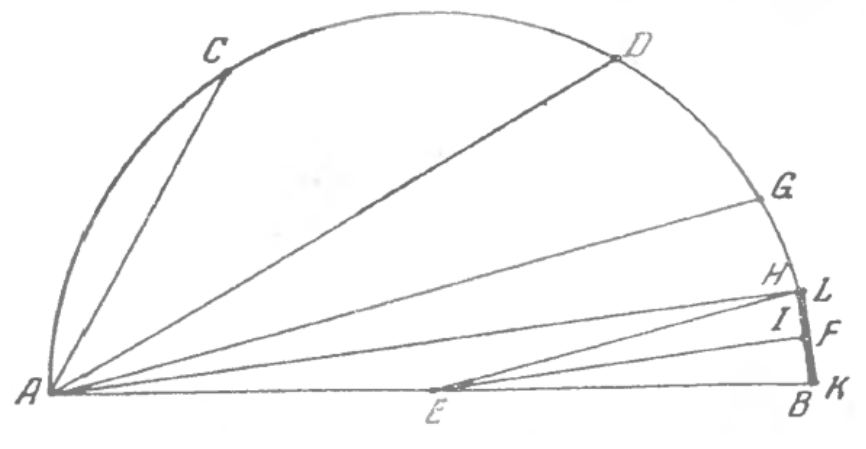

Fonte: al-Kāshī (1954, p.331).

4““...] угол АНВ прямой по тридцатому предложению третьей [книги] «Начал» $\left[{ }^{18}\right]$ и, следовательно, квадрат АВ равен квадратам AH, HB но «теореме невесты» $\left.{ }^{19}\right] . ”(A L-K \overline{A S H I} ; 1954$, p.329, tradução nossa). 


\section{Figura 5}

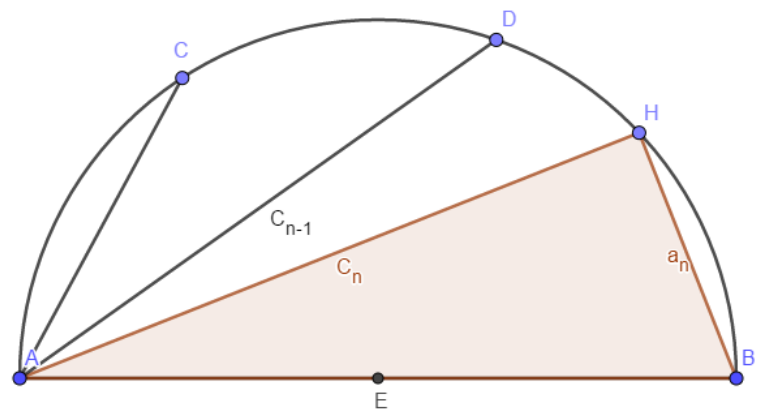

Fonte: Elaborado pela autora (2020).

Enquanto na primeira parte do tratado al-Kāshī utiliza quatro proposições de $O s$ Elementos de Euclides (proposição oito do livro seis, dezenove do livro sete, três do livro três e proposição trinta do livro três), na segunda, ele cita duas: a proposição quinze do quarto livro e a proposição trinta do terceiro livro, mas tomamos o cuidado de conferir em Euclides (2009) se as proposições citadas correspondiam a sua utilização e, verificamos que, tanto na primeira quanto na segunda parte do tratado, ao invés de ser a proposição trinta do livro três, o correto seria a proposição trinta e um do livro três, as outra estão corretas. Em nenhuma parte do Tratado da Circunferência al-Kāshī cita diretamente Os Elementos de Euclides, apenas fala a proposição e o livro, atribuímos isso ao fato de que era um livro extremamente difundido entre os estudiosos do mundo islâmico. Quando subentendido no texto que se trata de "Os Elementos", o tradutor Rozenfeld o escreve assim $\langle<O s$ Elementos $\rangle>$, entre $\langle<>>$. Já as demais palavras, atribuídas de acordo com o contexto, entre colchetes [ ].

Assim, desconsiderando a notação moderna, mas considerando o método obtido através de $c_{n}=\sqrt{R \cdot\left(2 R+c_{n-1}\right)}, n \geq 1$ e $a_{n}=\sqrt{4 r^{2}-c_{n}^{2}}, n \geq 1$, al-Kāsh̄̄ pôde calcular o perímetro do polígono regular inscrito à circunferência. Mas, como calcular o perímetro do semelhante circunscrito?

Consideremos agora, o seguinte trecho (Figura 6):

EI é a metade da linha $\mathrm{AH}$, uma vez que os triângulos $\mathrm{AHB}$ e EIB são semelhantes em virtude de que, os ângulos H e I são retos e o ângulo Â, na circunferência que acompanha o arco $\mathrm{BH}$, é igual ao ângulo central $\hat{\mathrm{E}}$, que 
Figura 6 - Ampliação de detalhe da figura 4.

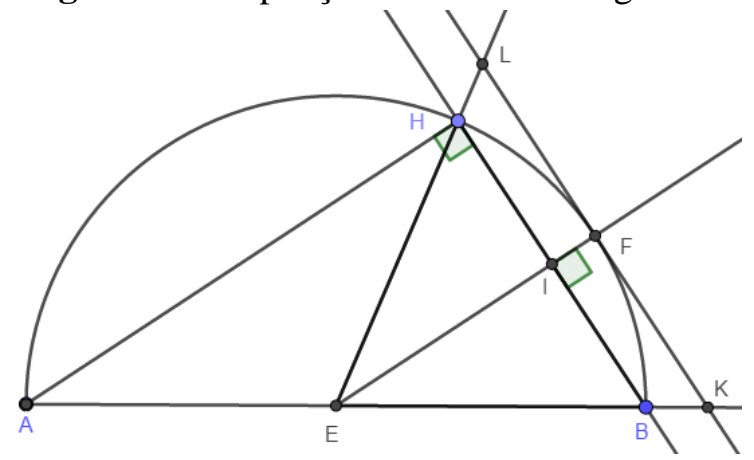

Fonte: Elaborado pela autora (2020).

Al-Kāshī usou Semelhança de Triângulos entre os triângulos $A H B$ e EIB juntamente com o fato de a reta suporte do segmento $K L$ tangenciar o ponto $F$ e, de acordo com o que conhecemos hoje como Teorema do Valor Médio, obteve que $\frac{\overline{E I}}{\overline{E F}-\overline{E I}}=$ $\frac{\overline{H B}}{\overline{K L}-\overline{H B}}$, onde $K L$ é o lado do polígono semelhante circunscrito e $\overline{E I}, \overline{E F}$ e $\overline{H B}$ são conhecidos. Mas, quantos lados devem ter esses polígonos regulares inscritos e circunscritos semelhantes?

\section{Parte 3: contando os lados do polígono regular inscrito}

Consideremos $\alpha_{n}, n \geq 0$ as medidas dos sucessivos arcos $A C, A D, A G, \ldots$, (Figura 4), observemos (Quadro 1):

$$
\text { Quadro } 1 \text { - Medida } \alpha_{n}, n \geq 1 \text { e } \alpha_{0}=\operatorname{arc}(A C)=60^{\circ} .
$$

\begin{tabular}{|c|}
\hline$\alpha_{1}=\operatorname{arc}(A D)=\operatorname{arc}(A C)+\frac{\operatorname{arc}(C B)}{2}=60^{\circ}+60^{\circ}=180^{\circ}-60^{\circ}=120^{\circ}$ \\
\hline$\alpha_{2}=\operatorname{arc}(A G)=60^{\circ}+60^{\circ}+30^{\circ}=\mathbf{1 8 0}^{\circ}-\frac{\mathbf{6 0}^{\circ}}{\mathbf{2}}=150^{\circ}$ \\
\hline$\alpha_{3}=\operatorname{arc}(A J)=60^{\circ}+60^{\circ}+30^{\circ}+15^{\circ}=180^{\circ}-\frac{60^{\circ}}{4}=\mathbf{1 8 0}^{\circ}-\frac{\mathbf{6 0}^{\circ}}{\mathbf{2}^{\mathbf{2}}}=165^{\circ}$ \\
\hline$\alpha_{4}=\operatorname{arc}(A P)=60^{\circ}+60^{\circ}+30^{\circ}+15^{\circ}+7,5^{\circ}=180^{\circ}-\frac{60^{\circ}}{8}=\mathbf{1 8 0}^{\circ}-\frac{\mathbf{2}^{3}}{\mathbf{2}^{3}}=172,5^{\circ}$ \\
\hline
\end{tabular}

5 “ЕІ есть половина [линии] АН, так как треугольники АНВ и ЕІВ подобны в силу того, что углы H, I прямые, угол А при окружности, стягиваемый дугой ВН, равен центральному углу Е, стягиваемому дугой ВF, являющейся половиной дуги ВН, а ЕВ есть половина АВ. Таким образом, ЕI есть половина AH.” (ROZENFELD, 1954, p.331, tradução nossa). 


\begin{tabular}{c}
$\alpha_{5}=\operatorname{arc}(A Q)=60^{\circ}+60^{\circ}+30^{\circ}+15^{\circ}+7,5^{\circ}+3,75^{\circ}=180^{\circ}-\frac{60^{\circ}}{16}=$ \\
$\mathbf{1 8 0}^{\circ}-\frac{\mathbf{6 0}^{\circ}}{\mathbf{2}^{\mathbf{4}}}=176,25^{\circ}$ \\
$\vdots$ \\
\hline $\boldsymbol{\alpha}_{\boldsymbol{n}}=\mathbf{1 8 0}^{\circ}-\frac{\mathbf{6 0}^{\circ}}{\mathbf{2}^{\boldsymbol{n}-\mathbf{1}}}$ \\
\hline
\end{tabular}

Fonte: Elaborado pela autora (2020).

Para o arco HB (Figura 6) juntamente com $\boldsymbol{\alpha}_{\boldsymbol{n}}=\mathbf{1 8 0}^{\circ}-\frac{\mathbf{6 0}^{\circ}}{2^{\mathbf{n - 1}}}, n \geq 1$ obtemos:

$$
\begin{gathered}
\operatorname{arc}(H B)=180^{\circ}-\operatorname{arc}(A D) \Rightarrow \\
\operatorname{arc}(H B)=180^{\circ}-\alpha_{n} \Rightarrow \\
\operatorname{arc}(H B)=180^{\circ}-\left(180^{\circ}-\frac{60^{\circ}}{2^{n-1}}\right) \Rightarrow \\
\operatorname{arc}(B)=\frac{360^{\circ}}{6.2^{n-1}} \\
\operatorname{arc}(H B)=\frac{1}{3.2^{n}} \cdot 360^{\circ}
\end{gathered}
$$

Portanto, o arco HG (Figura 6) corresponde a $\frac{1}{3.2^{n}}$ da circunferência. Logo, para obter o perímetro, basta multiplicar a medida da corda $H B=a_{n}$ pela quantidade de lados, 3. $2^{n}$, do polígono regular inscrito, assim, al-Kāshī obteve o polígono ideal.

\section{Conclusões e Considerações finais}

Neste trabalho, fizemos uma breve apresentação do Tratado da Circunferência de al-Kāshī e o seu contexto; explicitamos o principal resultado da primeira parte do tratado, o teorema de al-Kāshī. Sobre a segunda parte do tratado, fizemos o passo a passo de como al-Kāshī desenvolveu de maneira bastante engenhosa e surpreendente, utilizando o teorema por ele desenvolvido na primeira parte, um método para calcular o comprimento do polígono regular inscrito de $3.2^{n}$ lados e o seu semelhante circunscrito de maneira recursiva, através do que podemos representar hoje com as equações $c_{n}=$ $\sqrt{R \cdot\left(2 R+c_{n-1}\right)}$ e $a_{n}=\sqrt{4 r^{2}-c_{n}^{2}}, n \geq 1, c_{0}=\sqrt{3}$, de fundamental importância para a obtenção do comprimento da circunferência. Vemos também que a principal ferramenta para a execução das ideias de al-Kāshī foram os conhecimentos matemáticos presentes em Os Elementos de Euclides, nisto concluímos que ele dominava seu conteúdo sendo capaz de articular várias de suas proposições para obter um resultado inédito. $\mathrm{O}$ fato de 
Kaline França Correia Andrade

Um estudo da primeira e segunda parte do tratado da circunferência de Al-Kāsh̄̄

não enunciar diretamente $O$ s Elementos, mostra que este era extremamente difundido no contexto de al-Kāshī, bastando citar o livro e a proposição utilizada para que seus leitores o compreendessem.

Tanto a primeira como na segunda parte do Tratado da Circunferência apresentam diversos conceitos matemáticos compatíveis com o conteúdo matemático ensinado no nosso ensino básico: circunferência, semicircunferência, corda, arco, medida de ângulo, perpendicularidade, ponto médio, tangência, semelhança de triângulos, relações métricas na semicircunferência, recursividade, etc. Logo, diante de tudo que expusemos, concluímos que o Tratado da Circunferência de al-Kāshī apresenta potencialidades para o desenvolvimento da articulação entre história da matemática e ensino conforme citado em Saito e Dias (2013, p.96), algo que será mais explorado na continuação dos nossos estudos.

\section{Referências}

AL-KĀSHĪ, G. Al-Risāla al-Muhītīyya. Biblioteca Central de Āstāne Qudse Razawī. Coleção de matemática, n. 162, coleção geral n. 5389. Mashhad, Irã, 827 A.H./1424.

AL-KĀSHĪ, G. Traktat ob okruzhnosti. In: ROZENFELD, B. (trad.). Istoricomatematicheskie Issledovaniya, n.7, p.327-379, Moscou, 1954.

AZARIAN, M. Al-risāla al-muhītīyya: a summary. Missouri journal of mathematical sciences, v.22, n.2, p.64-85, 2010.

BELTRAN, M.; SAITO F.; TRINDADE, L. História da Ciência para formação de professores. São Paulo: Editora Livraria da Física, 2014.

EUCLIDES. Os Elementos. Tradução e introdução de Irineu Bicudo. São Paulo: Editora UNESP, 2009.

O'CONNOR, J.; ROBERTSON, E. Ghiyath al-Din Jamshid Mas'ud al-Kashi.

MacTutor. Um recurso online gratuito que contém biografias de matemáticos. School of Mathematics and Statistics University of St Andrews, Scotland, (1999). Disponível em: 〈https://mathshistory.st-andrews.ac.uk/Biographies/Al-Kashi/>. Acesso em: 27 fev.2021.

SAITO, F.; DIAS, M. Interface entre história da matemática e ensino: uma atividade desenvolvida com base num documento do século XVI. Ciência \& Educação (Bauru). Universidade Estadual Paulista Júlio de Mesquita Filho São Paulo, Brasil. v. 19, n. 1, p. 89-111, 2013. 